Case report

\title{
A Case Report of a Laparoscopic Operation for a Patient with Endometrial Cancer
}

\author{
Satoru Takeuchi \\ Kochi Health Sciences Center, Gynecology and Obstetrics, Kochi, Japan
}

\begin{abstract}
The purpose of this article is to describe our initial experience using laparoscopy to perform surgery for endometrial cancer. The patient was 59 years old with 2 para and was diagnosed as having a clinical endometrial adenocarcinoma in FIGO Stage Ia or Ib. Hysterectomy, bilateral salpingo-oophorectomy and lymph node sampling were performed using a laparoscopic procedure. We analyzed the problems and advantages of the procedure in this case.
\end{abstract}

Key words: laparoscopic surgery, endometrial cancer

(J Rural Med 2009; 4(1): 32-34)

\section{Introduction}

Laparoscopic operation has been said to be a less invasive surgery than laparotomy. Advanced laparoscopic procedures are increasingly being used as alternatives to laparotomy in gynecologic surgery. Laparoscopic operation for gynecologic malignancy is becoming a standard procedure worldwide. Howevere in Japan, laparoscopic operation for gynecologic malignancy has not prevailed. The purpose of this article is to describe our initial experience using laparoscopy to perform surgery for endometrial cancer. We analyzed the problems and advantages of the procedure in this case.

\section{Case Presentation}

The patient was 59 years old with 2 para. She visited a clinic because of genital bleeding. Histological examination revealed a grade 1 endometrioid adenocarcinoma. She was referred to our institution for treatment. When she visited

Correspondence to: Satoru Takeuchi

Kochi Health Sciences Center, Gynecology and Obstetrics, Ike 2125-1, Kochi City, Kochi 781-8555, Japan

E-mail: satoru_takeuchi@khsc.or.jp our institution, vaginal ultrasonograpy revealed a tumor that was $0.8 \mathrm{~cm}$ in diameter in the uterine cavity with adenomyosis in the uterine body (Figure 1). Biopsy revealed that it was a grade 1 endometrioid adenocarcinoma. The suspected tumor was identified in the uterine cavity in MRI images (Figure 2). No tumor or lymph node swelling was identified in the abdomen or chest via CT scan. According to the 2006 treatment guidelines for corpus cancer of the Japan Society of Gynecologic Oncology, hysterectomy and bilateral salpingo-oophorectomy (BSO) are recommended in patients with a stage Ia endometrial adenocarcinoma and a G1 endometrioid adenocarcinoma. Hysterectomy, BSO and retroperitoneal lymph node dissection are recommended for cases other than the above corpus cancer. The patient was diagnosed as having a clinical endometrial adenocarcinoma in FIGO Stage Ia or Ib with a G1 endometrioid adenocarcinoma. Hysterectomy, bilateral salpingo-oophorectomy and lymph node sampling via a laparoscopic procedure were planned with.

The surgical technique for the laparoscopic procedure was as follows. The procedure was performed under general endotracheal anesthesia. The patient was placed in the litho-

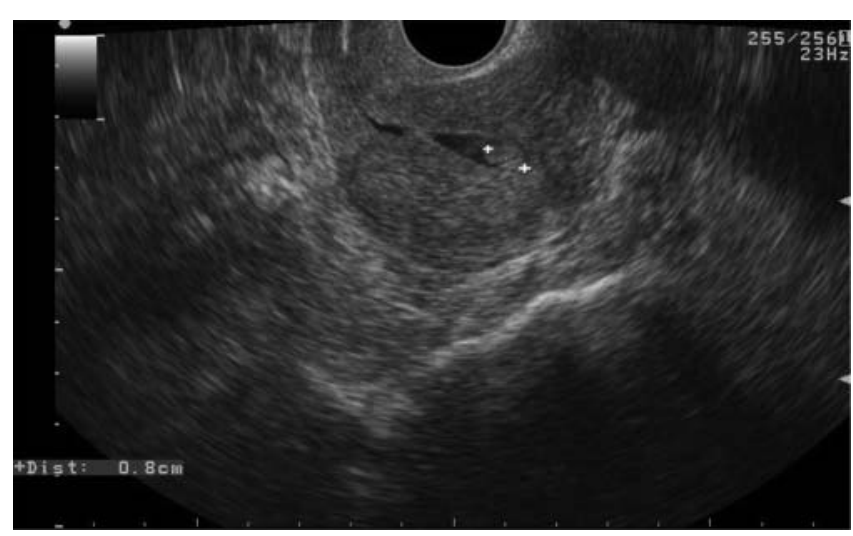

Figure 1 Tumor identified using vaginal ultrasonography. 


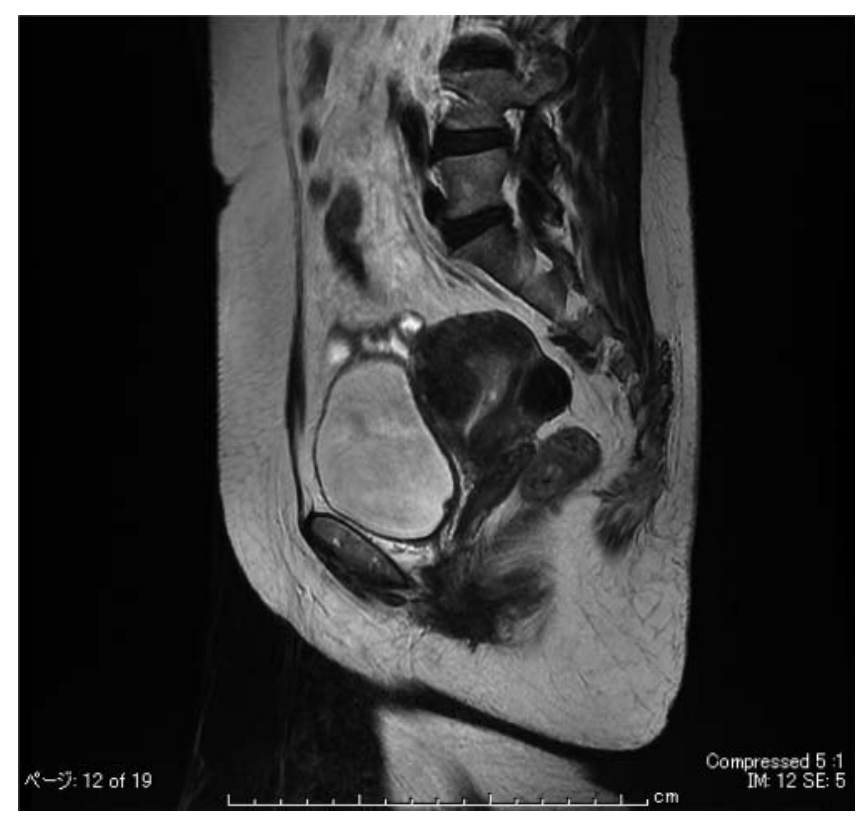

Figure 2 Sagittal T2 MRI image.

tomy position with her arms tucked at her sides. A Foley catheter was inserted in the bladder, and a vagi-pipe was placed in the vaginal vault. The patient was then placed in the steep Trendelenburg position. A 12-mm trocar was placed at the umbilicus, and a 12-mm laparoscopic scope was inserted. The abdominal cavity was insufflated. Two trocars $(5-\mathrm{mm})$ were then placed in the right and left lower quadrants, and an additional trocar (12-mm) was inserted in the middle between the pubic symphysis and the umbilicus. The pelvis and abdomen were explored to rule out intraperitoneal disease. The isthmus of the bilateral fallopian tubes were ligated with clips. Ascites was not seen. About $200 \mathrm{ml}$ saline solution was infused into the pelvic cavity and then retrieved for a Papanicolaou smear. An incision was made in the vesicouterine peritoneum and the peritoneum over the psoas muscle immediately lateral to the infundibulopelvic ligament. The round ligaments were transected bilaterally. The ureters were identified. The uterine artery and vein were identified and transected at the point of origin from the internal iliac vessels with a harmonic scalpel. The infundibulopelvic ligaments were transected bilaterally. The uterosacral ligaments were then transected. The bladder was mobilized inferiorly. The ureters were separated from their medial attachments to the peritoneum. The branches of uterine vessels to the ureter were dissected bilaterally. The ureter and uterine artery on the uterus side were separated completely. The overlying tissue of the ureters was dissected to the junction of the bladder. Paravaginal tissue just

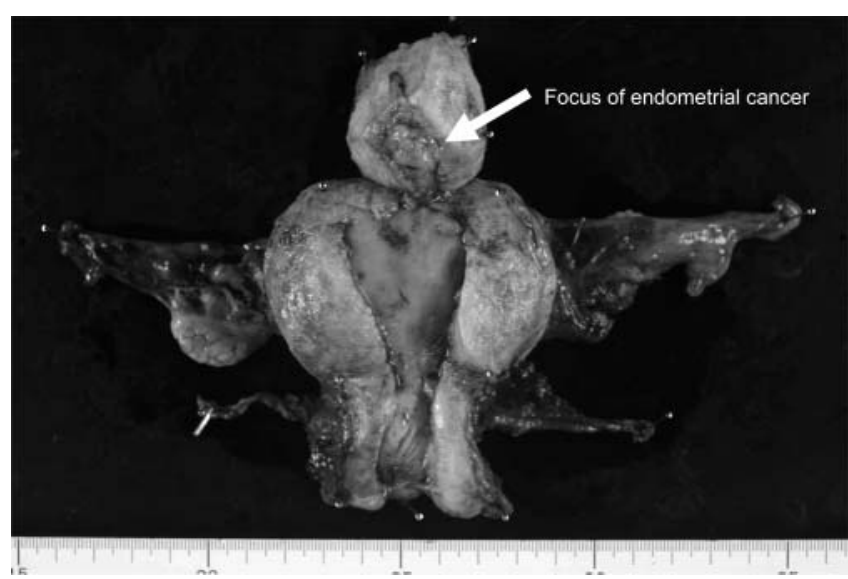

Figure 3 Resected specimen.

oral to the ureter were sutured, ligated and dissected bilaterally. The anterior vagina was incised with a monopolar electric scalpel, and then the rest of the vagina was incised with a harmonic scalpel. The specimen, including the upper vaginal margin, cervix and uterus, was completely separated from the upper vagina and removed via the vagina. The vaginal cuff was suture-ligated continuously with 1-0 Vicryl laparoscopically. The lymph-bearing tissue along the pelvis was then evaluated for obvious metastatic disease. The lymph-bearing tissue was removed from over the external iliac artery and vein, and the obturator space above the obturator nerve. After irrigating and ensuring hemostasis, a drainage tube that was $6-\mathrm{mm}$ in diameter was placed. The 12-mm ports were closed under direct visualization with 1-0 polyglycolic acid sutures.

The operation time was 5 hours 28 minutes. Blood loss was minimal. The patient did not require a blood transfusion. The total number of pelvic lymph nodes harvested was three. The post-operative course was uneventful. The patient was discharged on the sixth day after the surgery. In the resected specimen, a tumor about $2 \mathrm{~cm}$ in diameter was recognized in the fundus of the endometrium with no suspicious myometrial invasion macroscopically (Figure 3 ). Histological examination revealed the tumor to be slightly invading into the myometrium and to have any lymph node metastasis. The Papanicolaou smear test of the ascites was negative. The patient was in stage pT1bN0M0 with no lymph-vascular space involvement.

\section{Discussion}

Laparoscopic surgery has been increasingly used in malignant tumors. In the 1990's, gynecologic oncologists began using laparoscopic surgery to perform hysterectomy 
and lymph node dissection for treatment of patients with endometrial cancer ${ }^{1}$. Patients are increasingly aware of the option of minimally invasive surgery to treat endometrial cancer and often request this approach ${ }^{1)}$. Intrinsically, laparoscopic surgery has the characteristics of microscopic surgery and has the benefit of making it easy to perform meticulous procedures. An operator looks at the operating site from a distance of about $30-50 \mathrm{~cm}$ during the procedure of laparotomy. On the other hand, a laparoscope can reach any distance from the operation site and magnify the target tissue, and the operator can see behind the targeted organs. In our case, in performing resection of small vessels between the uterine artery and ureter, each vessel could be resected individually because of the similar characteristics of laparoscopic surgery compared to microscopic surgery and a viewpoint parallel to the ureter and uterine artery. The anterior vesicouterine ligament could be resected with clear organ separation and minimal blood loss. As for lymph node dissection, in the present case, three lymph nodes were retrieved. This number was insufficient for lymphadenectomy. We experienced laparoscopic collection of the pelvic lymph nodes and decided that several attempts should be performed, such as the extraperitoneal approach or setting the port of the scope on the upper abdomen to retrieve more pelvic lymph nodes, especially common iliac lymph nodes ${ }^{2}$. The procedure may not be performed as pelvic lymphadenectomy until pelvic lymph nodes are sufficiently retrieved and the total number of retrieved lymph nodes exceeds twenty.

The short-term results of laparoscopic surgery for endometrial cancer are equivalent or better than those achieved with open surgery, whereas the long-term results obtained by laparoscopic surgery and laparotomy seem equivalent, but more studies are needed assessing this outcome ${ }^{3)}$.

Although laparoscopic surgery has many advantages, it is also associated with a number of potential drawbacks, including limited range of motion intra-abdominally, counterintuitive movements, amplification of tremors in prolonged cases because of the length and rigidity of the instrumentation and reduced depth perception secondary to a two-dimensional view ${ }^{1)}$. Laparoscopic surgery has many advantages. Laparoscopic surgery is associated with less morbidity than laparotomy ${ }^{1)}$. Numerous studies have shown that laparoscopic surgery is associated with decreased blood loss, lower transfusion rates, decreased analgesic requirements, shorter length of hospital stay, improved cosmesis and faster return to normal daily activities ${ }^{1}$. In this case, blood loss was minimal. The characteristics of laparoscopic surgery are theoretically very useful in the operation for malignant tumors; however, the laparoscopic operation requires skill, especially in performing pelvic lymph node dissection. Laparoscopic operation is useful when the operator chooses adequate cases in accordance with his or her own level of skill in performing the procedure. Laparoscopic resection of the anterior vesicouterine ligament would be feasible, although sufficient training is required to attempt performance of laparoscopic pelvic lymphadenoctomy.

\section{References}

1. Ramires PT, Soliman PT, Schmeler KM, et al. Laparoscopic and robotic techniques for radical hysterectomy in patients with early-stage cervical cancer. Gynecol Oncol 2008; 110 : 21-24.

2. Dowdy SC, Aletti G, Cliby WA, et al. Extra-peritoneal laparoscopic para-aortic lymphadenctomy- A prospective cohort study of 293 patients with endometrial cancer. Gynecol Oncol 2008; 111: 418-424.

3. de la Orden SG, Reza MM, Blasco JA, et al. Laparoscopic hysterectomy in the treatment of endometrial cancer: a systematic review. J Minim Invasive Gynecol 2008; 15: 395 401. 